a broom, one a vacuum-cleaner and one a carpet-sweeper. Each janitor is better adapted than the others to collecting certain trash items, but there are many items that can be collected by any of several janitors. A good strategy for all janitors is to forage together, thereby constantly assuring themselves of trash-rich areas and avoiding areas partly cleared by other janitors.

For whatever reason species do forage together, it is striking that flock members of widely different families often resemble each other in appearance, vocalizations, or both. As examples of this co-evolutionary phenomenon, termed social mimicry, Moynihan ${ }^{19}$ noted that flocking tanagers in the mountains of Panama are mainly black and/or yellow, in the northern Andes brilliant blue or blue and yellow, and in the south-central Andes blue-grey dorsally and brown ventrally. A prime example of this tendency for birds of a feather to flock together is the 'brown and black flock' of New Guinea, first noticed by the flamboyant Italian explorer Count Luigi d'Albertis in 1880 and rediscovered much later by Bell ${ }^{20}$. The flock involves up to 22 medium-sized, omnivorous bird species from seven families (birds of paradise, babblers, whistlers, drongos, cuckooshrikes, honey-eaters and flycatchers). All these species are either mainly rufous or mainly black; in three, the male is black, the female rufuous. The flock is led by a group of the babbler Garritornis isidori, of which one individual is the actual leader and has a distincitive 'leader' call while his/her conspecifics have a 'follower' call. At least five other species in the flock mimic one or other of the babblers' calls.

Why should species from such different families converge in plumage? We are not dealing here with detailed mimicry of plumage patterns, but merely broad resemblances in overall colour. Moynihan points out that interspecific signals are required so that mixed flocks can form and maintain cohesion. The convergence in these signals may be for economy: it is much easier for a flock member to stay with the flock and avoid wandering off with some non-flock species if it need only remember to look for brown or black birds, instead of keeping 21 different colour patterns in mind.

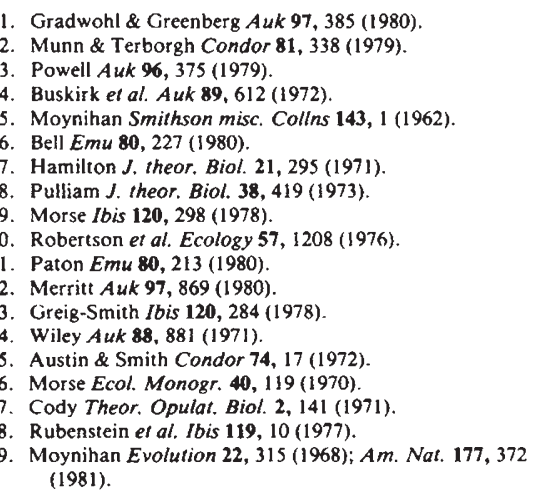

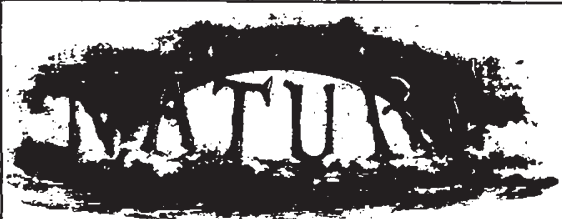

THE CHEMISTRY OF THE SUN

Although in the year 1866 a great many people were familiar with the spots on the sun, those who had been favoured by a sight of a total eclipse, and many more who had read the accounts of total eclipses, knew that there was a great deal more of the sun than one generally sees. From the time of Stannyan, who observed the prominences at Berne, down to the year 1842 , let us say, several eclipses had been observed, and very beautiful coloured phenomena had been recorded by different observes. Red things had been seen projecting round the dark moon during the time of eclipse, and although many held them to be beautiful effects produced by the passage of the moon over the sun, or even clouds in the atmosphere of the moon coloured by the strange way in which the solar light then fell upon them, a larger number of people, on the other hand, insisted that these things must really belong to the sun. Now if that were so, it was perfecly clear that we should not be contented with merely observing the chemical nature of the spots.

We have a round dark moon, which in this case is represented as entirely covering the sun; then these different prominences and luminosities, this wonderful set of streamers, or whatever you like to call them, which seem to veil, or to render less distinct, something else which islying beyond them. You will see here that some of these prominences are red, and others have a yellow tinge, and that, quite independent of the colour of the prominences, we have the most exquisite coloured effects. Sometimes the radial structure is not so marked, and reveals

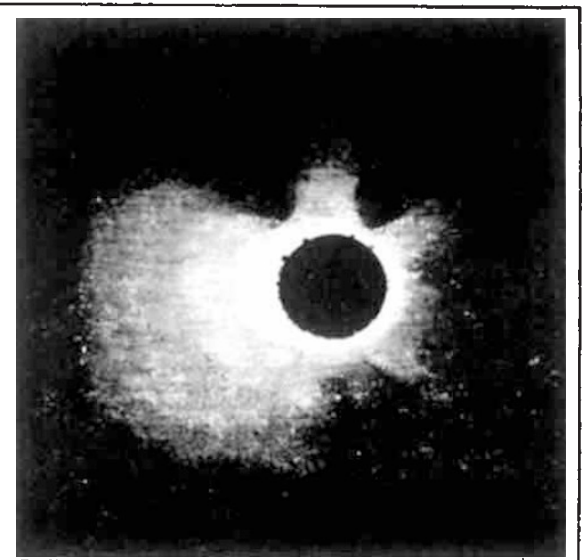

Eclipse of 1870 . Photograph of the corona taken at Syracuse.

indications of structure further away from the sun You see wonderfully delicate tracery, lines being seen now in one part and now in another. In the photograph taken during the eclipse of 1870 we see that the luminosity of the solar atmosphere was excessively irregular, by which I mean that in one part we get a very considerable excess of light, quite independent of the sharply defined prominences, whereas in other portions the atmosphere of the sun at the same height is not nearly so luminous. Now in none of these cases have we been able to see the thing which struck us most clearly the moment the artificial eclipse system was set at work. It is a good indication of the extreme difficulty of making observations during eclipses, and how important it is that one should have a method which makes us independent of them.

From Nature 24, 21 July, 271, 1881.

\title{
Origin of the galaxies
}

\section{from Joseph Silk}

COSMOLOGISTS generally believe that galaxies formed as a consequence of the growth of tiny density fluctuations in the very early Universe. The remarkable uniformity of the cosmic microwave background radiation has provided convincing evidence for the high degree of regularity of the Universe at an early epoch but within the past year two groups of astronomers, at Florence and Princeton, have reported evidence for a very weak anisotropy on an angular scale of $90^{\circ}$, which is probably produced by similar matter fluctuations to those from which the galaxies evolved.

One of the most tantalizing issues in cosmology concerns the origin of these fluctuations. It is thought that the initial conditions of the big bang might provide an answer. Perhaps the Universe is the way it is because of the way it was. Surely the resourceful cosmologist can improve on this hollow echo of creationism, for if the initial conditions were very different, then we would not be here to take stock of the situation. This concept of the observer's role in determining the state of the Universe has been elevated by Robert Dicke and Brandon Carter to the status of a fundamental cosmological principle: the anthropic principle. Its power arises

Joseph Silk is in the Astronomy Department at University of California, Berkeley. because the growth of fluctuations is inevitable from the beginning of time.

Consider the history of a fluctuation destined eventually to form a galaxy. It grew in amplitude throughout much of the early expansion of the Universe. The earliest epoch that the cosmologist can usefully discuss is the Planck instant, a mere $10^{-43}$ second after the singularity. At this time, our galaxy-sized fluctuation must have had an infinitesimal but nonzero density contrast. These inhomogeneities are best considered as fluctuations in the spatial curvature, or slight wrinkles in the geometry of space-time. The magnitude of such primordial wrinkles must be about 1 part in $10^{3}$, if fluctuations in the energy density are considered; for fluctuations in the matter component alone, the associated wrinkles attain a considerably smaller but well defined value. Were the fluctuation amplitudes much smaller, galaxies would not have formed by now; were they much larger, eventual collapse in the early Universe would have formed separate topologically disjoint universes, bearing little relation to our observed universe.

Although this argument purports to show that the initial conditions may have been unique, it certainly does not provide any explanation. One is left with the following conundrum. The very early 